\title{
শিলালিপিতে বাংলাদেশের ঐতিহ্য: হাজী খাজা শাহবাজ ও মরিয়ম সালেহা মসজিদের শিলালিপির উপর একটি বিশ্লেষণধর্মী আলোচনা
}

\section{Tradition of Bangladesh in Inscriptions: An Analytical Discussion on the Inscriptions of Haji Khwaja Shahbaz Mosque and Mariam Saleha Mosque}

\author{
ফারজানা আক্তার* এবং মুহাম্মদ শামসুজ্জামান**
}

সার সংক্ষেপ: ইতিহাস জাতির দর্গণ। মানব গোষ্ঠীর কার্যক্রম ইতিহাস পঠন-পাঠনের মধ্য দিয়ে জানা যায়, আর ইতিহাস পঠন-পাঠনের অন্যতম একটি গুরুত্বপূর্ণ উৎস হচ্ছে প্রত্মতাক্ত্বিক উৎস। কেননা প্রত্মতত্ত্ব কোনো ভূখণ্ডের অতীতে বিলুপ্ত হয়ে যাওয়া মানুভের জীবনের সাথে জড়িত সব ধরনের আর্থসামাজিক, রাজনৈতিক ও সাংস্কৃতিক নিদর্শনাদি অনাবৃত ও আলোচনার মাধ্যমে তাদের পূর্ণাঙ্গ ইতিহাস রচনার যাবতীয় সূত্রাদি সরবরাহ করে থাকে। ফলে লিখিত তথ্যের ইতিহাস যেখানে নির্বাক, সেখানে প্রত্নতত্তুই হয়ে উঠে সবাক। সে কারণে পণ্ডিতরা প্রত্নতত্ত্বকে ইতিহাস ও ঐতিহ্যের শেকড় বলে অভিহিত করেন। আর প্রত্মতত্তের অন্যতম উৎস হন্ছে শিলালিপি। এরকম দুটি উল্লেখযোগ্য শিলালিপি হচ্ছে হাজী খাজা শাহবাজ ও মরিয়ম সালেহা মসজিদের শিলালিপি। আলোচ্য শিলালিপি দুটিতে সমকালীন মুঘল বাংলার আর্থ-সামাজিক, রাজনৈতিক, ধর্মীয় ও সংস্কৃতির বস্তুনিষ্ঠ তথ্য প্রতিফলিত হয়েছে।

মূল শব্দসমূহ: ইতিহাস, সংস্কৃতি, প্রত্মতত্ত্ব, শিলালিপি এবং মসজিদ নির্মাণ।

\begin{abstract}
History is the mirror of humankind. The activities of human society are known through the reading of history, and the archaeological source is one of the important sources of reading history. Because Archeology provides all the materials for writing the complete history of the extinct people of the past by exposing and discussing all kinds of socio-economic, political and cultural discoveries involving their lives. As a result, where the history of written information is silent, there archeology reveals many things about the past. For this reason, scholars term Archeology as the root of history and tradition. And inscriptions are one of the sources of Archeology. Two such important inscriptions are those of Haji Khwaja Shahbaz Mosque
\end{abstract}

* ফারজানা আক্তার, লেকচারার, ইসলানের ইতিহাস ও সভ্যতা বিভাগ, এশিয়ান ইউনিভার্সিটি অব বাংলাদেশ, উত্তরা, ঢাকা। E-mail:farjanashakil86@gmail.com

** মুহাম্মদ শামসুজ্জামান, লেকচারার, ইসলামের ইতিহাস ও সভ্যতা বিভাগ, এশিয়ান ইউনিভার্সিটি অব বাংলাদেশ, উত্তরা, ঢাকা।

E-mail:samsuzzamanrajib@yahoo.com 
and Mariam Saleha Mosque. These two inscriptions reflect the realistic information of the socio-economic, political, religious and cultural scenario of the contemporary Bengal of the Mughal Period.

Keywords: History, Culture, Inscription and Mosque built.

ভূমিকা

ঐতিহাসিক উপাদান হিসেবে শিলালিপির গুরুত্ব অপরিসীম। এগুলো থেকে যে তথ্য পাওয়া যায় তাতে কোনো পক্ষপাতিত্ব বা অসত্য নেই। সমসাময়িক কালের ইতিহালের জন্য সেগুলো মূল্যবান তথ্য বহন করে। মধ্যযুগে বাংলাদেশে প্রাপ্ত শিলালিপিগুলো সাধারণত আরবি ও ফারসি ভাষার লেখা এবং এগুলো মসজিদ, মাদ্রাসা, খানকাহ, সমাধি, পুল এবং কুয়া ইত্যাদি নির্মাণ বা খননের সময় উৎকীর্ণ করা হত। এই শিলালিপিগুনোতে নির্মাতার নাম, নির্মাণ তারিখ, সমসাময়িক বাংলার আর্থ-সামাজিক, রাজনৈতিক ও সংস্কৃতির বস্তুনিষ্ঠ তথ্য প্রতিফলিত হয়েছে। ফলে সমকালীন মুসলিম সমাজ সম্পর্কে একটি ধারণা লাভের সুয্যাগ শিলালিপিসমূহ থেকে পাওয়া সম্ভব। মুঘল আমলে ঢাকায় ১৬৭৮-৭৯ খ্রিষ্টাব্দে নির্মিত হাজী খাজা শাহবাজ মসজিদের শিলালিপি (চিত্র১) এবং ১৭০৬ খ্রিষ্টাব্দে নির্মিত মরিয়ম সালেহা মসজিদের ফারসি ভাযায় উৎকীর্ণ শিলালিপি (চিত্র- ২) দু’টি নিয়ে বিস্তৃত কোনো গবেষণাকর্ম ইতিপূর্বে পরিচালিত হয়নি। ড. এ কে এম ইয়াকুব আলী তাঁর ‘মুসলিম স্থাপত্য ও শিল্পকলা’ গ্রন্থে ভারতীয় উপমহাদেশের মুঘল স্থাপত্য এবং বাংলার সুলতানী স্থাপত্য সম্পর্কে আলোচনা করলেও মুঘল আমলের উল্লেখযোগ্য এই দু’টি মসজিদ সম্পর্কে কোনো আলোচনা করেননি। আলোচনার সুবিধার্থে বক্ষ্যমান প্রবন্ধে প্রথনে হাজী খাজা শাহবাজ মসজিদের শিলালিপি ও পরে মরিয়ম সালেহা মসজিদের শিলালিপি সম্পর্কে বিস্তারিত তথ্য তুলে ধরা হবে।

\section{গবেষণা পদ্ধতি}

গবেষণার জন্য দু’ধরনের উৎস থেকে তথ্য সং্রহ করা হয়েছে। প্রাথমিক উৎস হিসেবে সরেজমনি পর্যবেক্ষণ ও নিজস্ব বিচার বিশ্লেষণ এবং সেকেন্ডারি উৎস হিসেবে বিভিন্ন গ্রন্থ থেকে তথ্য-উপাত্ত গ্রহণ করা হয়েছে।

\section{হাজী খাজা শাহবাজ মসজিদের শিলালিপি}

মসজিদটি ঢাকা রেসকোর্স ময়দান (সোহরাওয়ার্দী উদ্যান)-এর দক্ষিণ-পশ্চিম কোণে এবং তিন নেতার মাজারের ঠিক পূর্ব পার্শ্বে অবস্থিত। মসজিদটির বাহ্যিক আয়তন উত্তর-দক্ষিণে ২০.৭২ মিটার এবং পূর্ব-পশ্চিন্মে ৭.৯২ মিটার। অষ্টভূজাকৃতির কর্ণার বুরুজসমূহের শীর্ষদেশ পঞ্জর আকৃতির কিউপোলায় আচ্ছাদিত এবং ছাদের উপর বন্ধাকৃতির তিনটি গম্বুজ শীর্ষ কলস ফিনিয়েলে সজ্জিত। পূর্ব ফাসাদের তিনটি খাঁত খিলান বিশিষ্ট তিনটি প্রবেশ পথের মধ্যবর্তটি গতানুগতিক রীতিতে অপেক্ষাকৃত বৃহদাকৃতির ও সামনের দিকে উদগত এবং এই উদগত কেন্দ্রীয় প্রবেশ পথটি আবার টারেট দ্বারা বেষ্টিত। কেন্দ্রীয় প্রবেশ পথের ন্যায় কেন্দ্রীয় মিহরাবটি পিছনের দিকে উদগত এবং উদগত অংশটির উভয় পার্শ সরু টারেট দ্বারা সজ্জিত। আগ্রা দুর্গ অভ্যন্তরস্থ সম্রাট শাহজাহানের খাস মহল এবং দিল্লী দুর্গ অভ্যন্তরে সম্রাট আওরঙ্গজব নির্মিত মতি মসজিদ (১৬৬২) এর অনুকরণে দেয়ালে প্রোথিত যুগল স্তম্ত শীর্ষ থেকে উখিত দুইটি বৃহদাকৃতির সুশমামণ্ডিত বহু খাঁজ বিশিষ্ট খিলান দ্বারা মসজিদ অভ্যন্তর তিনটি সম আয়তনের বর্গাকার অংশে বিভক্ত। এই বহু খাঁজ সম্বলিত খিলান এবং দেয়ালে প্রোথিত যুগল স্তম্ভসমূহ 
মসজিদ অভ্যন্তরের সৌন্দর্য বর্ধনে গুরুত্বপূর্ণ অবদান রেখেছে। শিলালিপিটি পাওয়া গেছে মসজিদটির প্রধান প্রবেশপথের ঠিক উপরে পূর্ব ফ্যাসাদে। উৎকীর্ণ করার সময় এর যে রূপ ছিল তা এখনও অক্ষুণ্ন রয়েছে। শিলালিপিটি কৃষ্ণ পাথরে অত্যন্ত সুন্দরভাবে নাসতালিক রীতিতে ফারসি ভাষায় খোদাই করা হয়েছে। দুটি আস্ত পাথর জোড়া দিয়ে এক লাইনে লিখিত এই শিলালিপিটির পরিমিতি ১88"X ১২শব্দ। শিলালিপিটি ৪টি অংশে বিভক্ত।

নিল্নে এই মসজিদের শিলালিপিটির ফার্সি উচ্চারণ বাংলা ভাষায় তুনে ধরা হল-

“ছখ়ত হাজি খাজো শাহবাওজ ইন বানাও ইয়ে পাকর- অনকে দার রাফ্আত বে আরশে আজম আমবাজ ওমাদেবার জবান আকল কুল তারিখে অন বনিয়াদে পাক-মাসজিদে জিব জাহাজি খাজা শাহবাওজ ওমাদে সানাহ্ ১০b-৯ হিজরি”।

শিলালিপিটির পাঠোদ্ধার করেছেন খ্যাতনামা ইতিহাসবিদ ড. আব্দুল করিম। ${ }^{8}$ তাঁর “Corpus of the Arabic and Persian inscriptions of Bengal” নামক গ্রন্থে শিলালিপিটির ইংরেজি অনুবাদ করা হয়েছে নিম্নরূপ:

Haji Kawajah Shabaz built this holy building, which may be said to rise to the throne of Allah. Tell, by tongue that knows all things that the date of this building comes from "This is the mosque of Haji Kawajah Shabaz"; the year 1089 A. H. (1678-79 A.D.)

বাংলা অর্থ-

‘হাজী খাজা শাহবাজ এই পবিত্র ইমারতটি নির্মাণ করেছেন । বলা যেতে পারে যে এটা আল্লাহর দরবারে পৌঁছেছে (আল্লাহর দরবার পর্যন্ত উঁচু)। বল, সেই জিহবার মাধ্যমে যা সব জানে, এই পবিত্র ইমারতটির তারিখটি এসেছে, “এই সুন্দর হাজী খাজা শাহবাজ মসজিদ”; ১০৮-৯ হিজরি বছরে (১৬৭৮-৭৯ খ্রি.)।

ব্যাখ্যা-

শিলালিপিটিতে সাধারণ বর্ণনা আছে এবং তা অনেকটা ফারসি কবিতার মত। ৪টি অংশের প্রথম অংশে দেখা যাচ্ছে মসজিদটির নির্মাতার নাম স্পষ্ট করে বলা হয়েছে হাজী খাজা শাহবাজ। তাঁর পরিচয় সম্পর্কে বাংলাপিডিয়া নামক কোবগ্রন্থে বলা হয়েছে, শাহজাদা মোহাম্মদ আজমের সুবাহদারিকালীন সময়ে ঢাকার বণিক খাজা শাহবাজ ১৬৭৯ খ্রিষ্টাব্দে এটি নির্মাণ করেন।৫ আর এর মাধ্যমে বুঝা যায়, লে সময়ে স্থানীয় লোকজন ধর্মীয় ইমারত নির্মাণ করত। তাদের অর্থনৈতিক স্বচ্ছলতা ছিল এবং সমাজে ব্যবসা-বাণিজ্যের প্রচলন ছিল। ইতিহাস সাক্ষ্য দেয় যে, মুঘল আমলে ঢাকা অর্থনৈতিকভাবে সমৃদ্ধ ছিল। আর এই সমৃদ্ধির সূচনা হয় ১৬১০ সালে ইসলাম খান কর্তৃক ঢাকায় রাজধানী স্থাপনের মাধ্যনে। ফনে ঢাকা ব্যবসায়ী ও বণিক শ্রেণির দৃষ্টি আকর্ষণ করতে সক্ষম হয়। সেই সময় থেকে ঢাকার অগ্রগতি এবং বাণিজ্য কেন্দ্র হিসেবে ঢাকার গুরুত্ব এতই বৃদ্ধি পায় যে, ওুধুমাত্র ব্যক্তিব্যবসায়ী ও ব্যাংকারগণ ঢাকায় আসেননি, বরং সংগঠিত ইউরোপীয় কোম্পানীসমূহও ঢাকায় কারখানা স্থাপন করেছিল এবং মুঘলদের এই পূর্বাঞ্চলীয় রাজধানীর সাথে খুবই জমজমাট এবং লাভজনক ব্যবসা চালিয়ে গিয়েছিল । সেবাস্টিয়ান ম্যানারিক একজন পুর্তগীজ ধর্মयাজক ছিলেন এবং ১৬৪০ সালে ঢাকায় এসেছিলেন। তিনি লিখেছেন যে, এই শহরে বিভিন্ন পণ্যের ব্যাপক ব্যবসা ও বাণিজ্যের সুবিধা থাকার কারণে বহু বিচিত্র জাতির ব্যবসায়ীরা এখানে এসে ভীড় জমাতো। ঢাকা বহু রকমের পণ্য রপ্তানি করত যার মধ্যে সবচেয়ে 
গুরুত্বপূর্ণ ছিল বয়নজাত পণ্য। এ পণ্যটি সমগ্র বঙ্গীয় প্রদেশের অর্থনৈতিক কার্যকলাপের সবচেয়ে গুরুত্বপূর্ণ হয়ে উঠেছিল। সুতি বস্ত্রের উৎপাদন এবং সারা বিশ্বের রপ্তানির কারণে ঢাকা সুখ্যাতি অর্জন করেছিল।

নামের সাথে ‘হাজী’ শব্দটির ব্যবহার দেখে বুঝা যায় সেই সময় সমাজে হজ্জ পালনের রীতি প্রচলিত ছিল এবং মানুষ ধর্ম পালন করত। বাংলাদেশে এখনও মানুষ হজ্জ করে আসলে তাঁর নানের পূর্বে হাজী শব্দটির ব্যবহার দেখা যায় এবং তাঁকে সমাজে অত্যন্ত সম্মানের দৃষ্টিতে দেখা হয়।

দ্বিতীয় অংশে ইমারতটি নির্মাণের কারণ প্রচ্ছন্নভাবে ফুটে উঠেছে। বলা হয়েছে “আল্লাহর দরবারে গৃহীত হয়েছে”। অর্থাৎ আল্লাহর সন্তুষ্টি অর্জনের জন্য ইমারতটি নির্মাণ করা হয়েছে এবং নির্মাতা আল্লাহর সন্তুষ্টি অর্জনের ব্যাপারে আশাবাদী। ধর্মীয় ইমারত তথা মসজিদ নির্মাণের ফজিলত পবিত্র কুরআন এবং হাদিসে বিবৃত হয়েছে যা মুসলমানদেরকে এ ধরনের কাজে উৎসাহী করে। পবিত্র কুরআনে বলা হয়েছে- “মসজিদসমূহ আল্লাহ্ তায়ালাকে স্মরণ করার জন্য। অতএব, তোমরা আল্লাহ্ তায়ালার সাথে আর কাউকে ডেকো না”।

এ সম্পর্কিত হাদিসসমূহ হল:

ইয়াহৃইয়া ইবৃন সুলায়মান (র).... ‘উবায়দুল্লাহ খাওলানী (র) থেকে বর্ণিত, তিনি উসমান ইব্ন আফ্ফান (রা)কে বলতে শুনেছেন, তিনি যখন মসজিদে নববী নির্মাণ করেছিলেন তখন লোকজনের মন্তব্যের প্রেক্ষিতে বলেছিলেন: তোমরা আমার উপর অনেক বাড়াবাড়ি করছ অথচ আমি রসুল সা.-কে বলতে শুনেছি: যে ব্যক্তি মসজিদ নির্মাণ করে, বুকায়র (র) বলেন: আমার মনে হয় রাবী ‘আসিম (র) তাঁর বর্ণনায় উল্লেখ করেছেন, আল্লাহর সন্ত্তির উদ্দেশ্যে, আল্লাহ তায়ালা তাঁর জন্য জান্নাতে অনুরূপ ঘর তৈরি করবেন। ১০

মাহমুদ ইবনে লাবীদ থেকে বর্ণিত। (তিনি বনেছেন) উসমান ইবনে আফ্ফান (রা) মসজিদ নির্মাণ করতে মনস্থ করলে লোকজন তা পছন্দ করলো না। বরং মসজিদ যেমন আছে তেমন রেখে দেয়াই তারা ভালো মনে করলো। তখন উসমান বললেন: আমি রসুল সা.-কে বলতে শুনেছি নিছক আল্লাহর সস্তুষ্টির উদ্দেশ্যে কেহ মসজিদ নির্মাণ করলে আল্লাহ তায়ালাও তাঁর জন্য বেরেশতের মধ্যে অনুরূপ একখানা ঘর তৈরি করেন ।১

এই হাদিস সম্পর্কে সে সময়ের মুসলমানরা সচেতন ছিল এবং এ ধরনের নির্মাণে যে অশেষ সওয়াব পাওয়া যায় তা তারা অত্যন্ত গুরুত্বের সাথে বিবেচনা করতো। হাজী খাজা শাহবাজ মসজিদের শিলালিপিটি লে কথাই প্রমাণ করছে।

শিলালিপিটিতে সন (১০b-৯ হিজরি) স্পস্টভাবে উল্লেখ আছে। আর এর ফলে মসজিদটির নির্মাণ কাল নিয়ে কোনো সংশয় নেই। এই সময়ে ভারতবর্ষ্যে মুঘল শাসন প্রতিষ্ঠিত ছিল এবং মুঘল সম্রাট ছিলেন আওরঈজেব। মসজিদটির সন এবং স্থাপত্য বৈশিষ্ট্য (যেমন: ৩ গম্বুজ) দেখলে প্রতীয়মান হয় যে, এটি সম্রাট আওরঙজেবের আমলে নির্মিত একটি মুঘল মসজিদ।

শিলালিপিটিতে কোনো শাসকের নাম নেই। এর পেছনে দুটি কারণ উল্লেখ করা যায়। প্রথমত, মুঘল সম্রাটগণ শাসনযন্ত্রকে বিকেন্দ্রীকরণ করেছিলেন। যেখানে কেন্দ্রের ন্যায় প্রদেশগুলো অনেকক্ষেত্রেই স্বাধীনতা ভোগ করত। দ্বিতীয়ত, মুঘল সম্রাটগণ শাসনকার্যে উদারতা প্রদর্শন করেছিলেন। তারা এসব নির্মাণ কাজে হস্তক্ষেপ করতেন না। তাদের মনোভঙ্গি ছিল বিশ্বজনীন। বাদশাহকে আনুগত্য প্রদান করলে বিশ্বের যে কেহ তার রাজ্যে বসবাস করতে পারত, এমনকি রষ্ট্রীয় দায়িত্বও লাভ করতে পারত। ১২ 
সুলতানী আমলের শিলালিপিগুনোতে ‘বিসমিল্লাহ’ ও হাদিলের ব্যবহার যতটা পাওয়া যায় মুঘল আমলের শিলালিপিতে ততটা দেখা যায় না। সুলতানি আমলের ১৫টি শিলালিপিতে ১১ বিসমিল্লাহ’র ব্যবহার দেখা যায়, ১. সিয়ান শিলালিপি, বীরভূম, পশ্চিম বঙ্গ, ভারত; ২. মুগিশ আল দ্বীন উজবেকের শিলালিপি (১২৫০-১২৫৭ খ্রি.), শীতল মাঠ নওগাঁ; ৩. মহাস্থানগড় শিলালিপি, বগুড়া; ৪. সিকান্দার শাহের ভাগলপুর শিলালিপি, বিহার, ভারত; ৫. সুলতান জালালউদ্দিন মোহাম্মদ শাহের সুলতানগঞ্জ শিলালিপি, গোদাগারি, রাজশাহী; ৬. পরবর্তী ইলিয়াস শাহী সুলতান নাসির আল-দ্বীন মাহমুদ শাহ (১৪৩৬-৫৯ খ্রি.), আমলের নারিন্দা শিলালিপি, ঢাকা; ৭. চিহিল গাজি সমাধি, দিনাজপুর; b. গরজরিপা শিলালিপি, শেরপুর; ৯. মুঙ্গের শিলালিপি, বিহার, ভারত; ১০. আলাউদ্দিন হোসেন শাহের সময় সূফী শাহ জালালের নির্দেশে খালিজ খানের সিলেট শিলালিপি; ১১. আলাউদ্দিন হোসেন শাহের সময়ে ত্রিবেনীর দুটি শিলালিপিতে ‘বিসমিল্লাহ’ পাওয়া গেছে (হুগলি, পশ্চিম বঙ্গ, ভারত); ১২. জয়পুরহাট, বগুড়া, কাইতা হার, শিলালিপি (আলাউদ্দিন হোসেন শাহের সময়ে ৯১৬ হিজরিতে নির্মিত মসজিদ); ১৩. ছোট সোনা মসজিদের শিলালিপি, ফিরোজপুর, নবাবগঞ্; ১৪. সাদিপুর শিলালিপি, মালদহ, পশ্চিমবঙ, ভারত (আলাউদ্দিন হোসেন শাহের সময়); ১৫. মুঙ্গের শিলালিপি, বিহার, ভারত (হোসেন শাহী সুলতান নাসির শাহের সময় নির্মিত একটি মসজিদে শিলালিপিতে পাওয়া গেছে)।

মুঘল আমলের ১১টি শিলালিপিতে১৪ ‘বিসমিল্লাহ’ পাওয়া গেছে, ১. মুঘল সম্রাট জাহাঙ্গীরের আমলে পশ্চিম বঙ্গের নেদিনীপুরের কেসিয়ারি গ্রামের একটি হাম্মাম খানার শিলালিপিতে; ২. সম্রাট শাহজাহানের সময় শেরপুরে (বীরভূম) নির্মিত একটি মসজিদের শিলালিপি; ৩. সম্রাট শাহজাহানের সময় পশ্চিমবঙ্গের মেদেনীপুরের হিজলী একটি মসজিদের শিলালিপি; 8. সম্রাট শাহজাহানের সময় আসামের হাজো তে পোয়া মেক্কা মসজিদের শিলালিপি; ৫. সম্রাট আওরঙ্গজেবের সময় পাদুয়া (মালদহ, পশ্চিমবঙ্গ, ভারত) শেখ জালাল আল-দ্বীন তাবরিজির দরগাহর শিলালিপি; ৬. সম্রাট আওরঙ্গজেবের শাসনামলে কসবা, শাহপুর (মালদহ, পশ্চিমবঙ্গ, ভারত) মসজিদের শিলালিপি; ৭. সম্রাট আওরঙজেবের শাসনামলে কেসিয়ারি (মেদিনীপুর, পশ্চিবঙ্গ, ভারত) মসজিদের শিলালিপি; b. সম্রাট শাহআলম বাহাদুর শাহের সময় ঢাকায় নতুন পল্টন মসজিদের শিলালিপি (আজিমপুর কবরস্থানের পশ্চিনে); ৯. সম্রাট মোহাম্মদ শাহের সময় ছোট পাদুয়া কুতুব শাহীর মসজিদ (হুগলি, পশ্চিমবঙ, ভারত); ১০. সম্রাট মোহাম্মদ শাহের সময় সুতালোরী, বরিশালের একটি মসিজদ; ১১. সম্রাট মোহাম্মদ শাহের সময় নোয়াখালীর বেগমগঞ্জ থানার বজরা মসজিদের শিলালিপি। ফলে দেখা যায় সুলতানী আমলের ন্যায় মুঘল আমলেও সমাজে ধর্মীয় আবেগ জারী ছিল।

আরো একটি বিষয় লক্ষণীয় শিলালিপির লেখাগুলো খুব ভালোভাবে বিন্যাস করা হয়েছে। অনেকটা ফারসি কবিতার মতো। যেমন: শিলালিপির দ্বিতীয় ও চতুর্থ অংশ শেষ হয়েছে ‘ওমাদ্দে’ শব্দ দ্বারা আবার প্রথম ও তৃতীয় অংশ শেষ হয়েছে ‘পাক’ শব্দ দ্বারা। অর্থাৎ ছন্দ তাত্ত্বিক একটা মিল লক্ষ্য করা যায় লেখাগুলোতে। সেই সাথে লেখাতে কোনো ভুল নেই। এতে বুঝা যায়, যেসব লিপিশিল্পীগণ এগুনো লিখত তারা দক্ষ ও শিক্ষিত ছিল এবং শ্দে ফারসি উচ্চারণ, পঠন ও পাঠন তাঁদের রপ্ত ছিল। অর্থাৎ সমাজে প্রাতিষ্ঠানিকভাবে ফারসি শেখার প্রচলন ছিল। ইতিহাস সাক্ষ্য দেয়, মুঘল বিজয়ের পর বাংলাদেশে ফারসি ভাষা, জ্ঞান চর্চা খুব প্রসার লাভ করেছিল। সম্রাট আকবরের দিওয়ান টোডরমল কর্তৃক সরকারি কাজ কর্মে ফারসি ভাষা ব্যবহারের প্রচলন করার পর থেকে বাঙালি হিন্দু-মুসলমান উভয় সম্প্রদায়ের মধ্যে ফারসি চর্চার ও এর উন্নতির প্রতি জোর পড়েছিল ১৫ 


\section{মরিয়ম সালেহা মসজিদের শিলালিপি}

মসজিদটি ঢাকা নিউ মার্কেটের পূর্বপাশে নিলক্ষেত বাবুপুরা এলাকায় অবস্থিত। অপরিকল্পিত মেরামত ও সম্প্রসারণ কার্যের ফলে মসজিদের আদি বৈশিষ্ট্য অনেকাংশেই বিলীন হয়ে গেছে। উত্তর-দক্ষিণে লম্বা এই মসজিদের আয়তন (ভিতরের দিকে) ১১.৮১১ মিটার x ৩.৯৮ মিটার। পূর্ব দেয়ালে আছে তিনটি প্রবেশপথ। এগুলি অর্ধগম্বুজাকৃতির খিলানযুক্ত। সামনের দেয়াল প্যানেল দ্বারা অলঙ্কৃত ও কেন্দ্রীয় অংশ বাইরের দিকে কিছুটা উদগত। দরজা বরাবর ভিতরে পশ্চিম দেয়ালে একটি করে দরজা আছে। চারকোণে চারটি অষ্টভুজাকৃতির মিনার বা টারেট আছে। ছাদের উপরে আছে তিনটি গম্বুজ। কেন্দ্রীয় গম্বুজ অন্য দু’টির চেট়ে বড়। ১৬ মুঘল স্থাপত্যের নিদর্শন বহনকারী এ মসজিদের একটি শিলালিপি রয়েছে মসজিদের কেন্দ্রীয় প্রবেশ পথের উপরে পূর্ব ফাসাদে। শিলালিপিটি এক খণ্ড কৃষ্ণ পাথরে নাসতালিক রীতিতে ফারসি ভাযায় খোদাই করা। শিলালিপিটি একটি বর্ডার দ্বারা সমান দুটি অংশে বিভক্ত, যেখানে তিনটি করে মোট ছয়টি লাইন রয়েছে এবং এর পরিমিতি ৬০ X ৩০ সে. মি.। বাংলা ভাষায় শিলালিপিটির ফার্সি উচ্চারণ নিম্নরূপ:

"মারিয়াম ইন মাসজেদ কে বেনোকারদে-তো কে দুনিয়াও ইয়ে ভাফা কারদে-ছলে তারিখে ফাওথ ফা মাসজিদে উ- খওছতাম খতে ফি কারদে-গোফত ইন মাসজেদ ওয়াছিএ জামান-মারিয়াম সালেহে বেনো কারদে-ছানাহ্ ১১১৮ হিজরি"

শিলালিপি বিশেষজ্ঞ শামসুদ্দিন আহমদ’, ড. আবদুল করিম১ প্রমুখ তাঁদের গ্রন্থে এই শিলালিপিটির নাম উল্লেখ করেননি। ড. আহমদ হাসান দানি শুধুমাত্র মসজিদটির তারিখ উল্লেখ করেছেন। ১৯ মোহাম্মদ ইউসুফ সিদ্দিকী তাঁর গ্রন্থে শিলালিপিটির খুব সংক্ষিপ্ত বর্ণনা তুলে ধরেছেন ২০ ঢাকা বিশ্ববিদ্যালয়ের ইসলামের ইতিহাস ও সংস্কৃতি বিভাগের প্রফেসর ড. হোশাররফ হোসাইন ভূঁইয়া তাঁর "The Mosque of Mariam Saleha" আর্টিকেলে শিলালিপিটির ইংরেজি ভাযায় পাঠোদ্ধার করেছেন। নিঙ্নে তা তুলে ধরা হল:

"Mariam built this palace of prostration and left this unfaithful world. When I looked for the date of the year of her demise as well as the mosque (constructed by her), an unseen voice revealed it, saying 'This major mosque (of the age) was built by Mariam Saleha' in the year 1118 A.H. (1706 A. D.)"

বাংলা অর্থ-

“মরিয়ম এই পবিত্র মসজিদ নির্মাণ করেছেন অবিশ্বস্ত দুনিয়া ছেড়ে দিয়ে। এই মসজিদের তারিখ এবং তাঁর মৃত্যুর তারিখ যখন আমি দেখি বলা হচ্ছে এই প্রশস্ত মসজিদটি সেই সময়ের বড় মসজিদ। মরিয়ম সালেহা এটা নির্মাণ করেছেন ১১১৮- হিজরিতে (১৭০৬ খ্রি.)”।

ব্যাখ্যা:

শিলালিপিটিতে কতকগুলো দিক সুস্পষ্ট। প্রথমত নির্মাতার নাম এখানে বলা হচ্ছে। অর্থাৎ তাঁর নামকে এখানে গুরুত্ব দেয়া হচ্ছে। তৎকালীন সময়ে শিলালিপিতে একজন মহিলার নাম চলে আসা অত্যন্ত গুরুত্বপূর্ণ। এতে বুঝা যায় সমাজে তিনি অত্যন্ত প্রভাবশালী ছিলেন। তাঁর পরিচয় সম্পর্কে প্রখ্যাত ঢাকা গবেষক হাকিম হাবিবুর রহমান 
বলেছেন যে, সম্রাট আওরঙ্গজেবের সময়ের ঢাকার মগবাজারের একজন বিখ্যাত সুফি শাহ নূরী গোলন্দাজের বোন ছিলেন মরিয়ম সালেহা ।২ প্রফেসর মো: আব্দুল্লাহ বলেন, মরিয়ম শেখ আব্দুল্লাহ মোজাল্দেদীর কন্যা এবং শেখ গোলাম নোহাম্মদ মোজাল্দেদীর নাতনী ছিলেন । ${ }^{20}$ মরিয়ম সালেহার বংশ লতিকা নিম্নরূপ: ${ }^{28}$

হযরত শাহ্ সৈয়দ গোলাম মো: মোজাদ্দেদী ওরফে বাঘু দেওয়ান (রহ.)

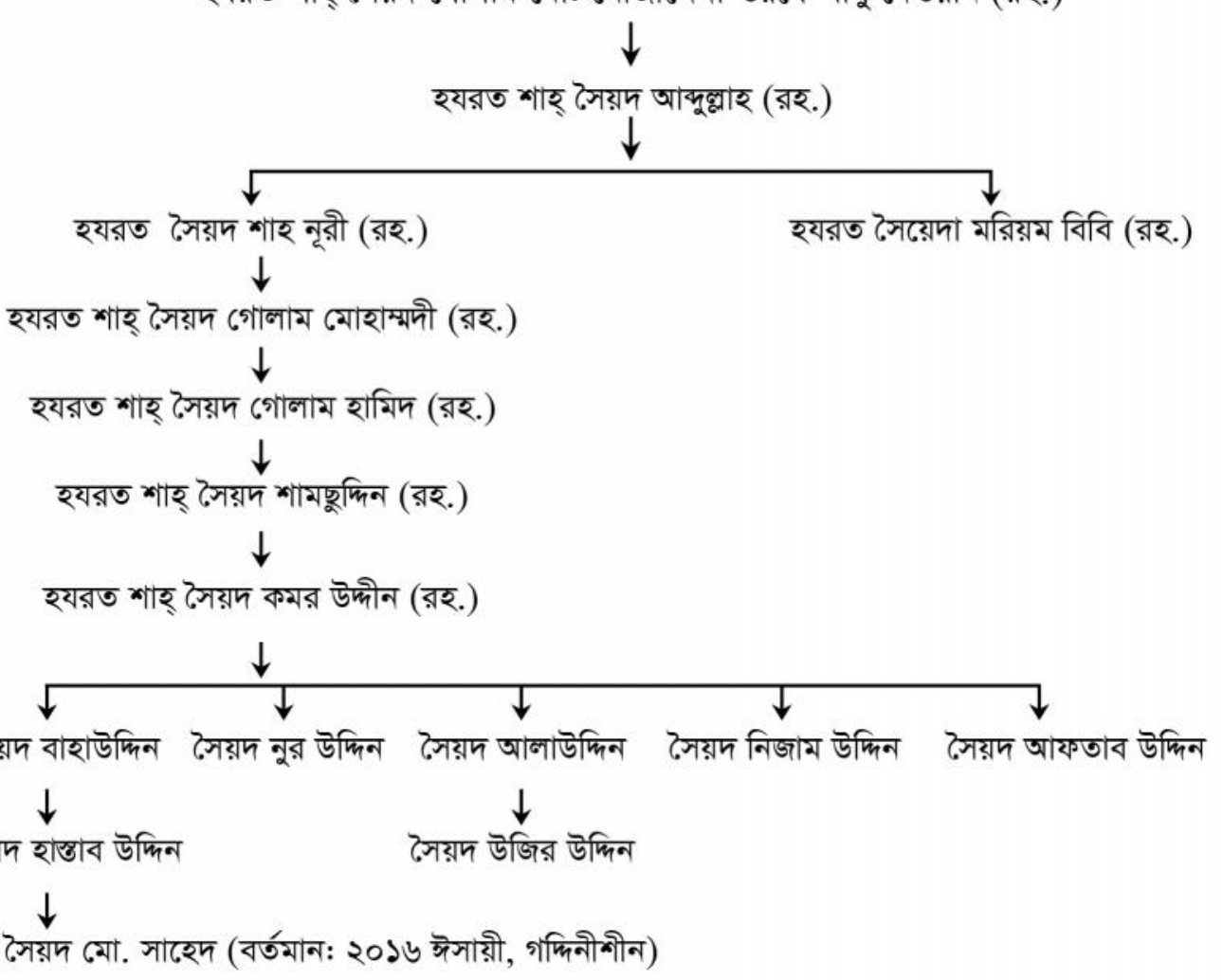

দ্বিতীয়ত, প্রথমে সাধারণভাবে বলা হচ্ছে মরিয়ম এটা নির্মাণ করেছেন কিন্তু পরে আবার বলা হচ্ছে মরিয়ম সালেহা এটি নির্মাণ করেছেন। সালেহা অর্থ ‘সৎকর্ম পরায়না’। কথিত আছে যে, তিনি ছিলেন অত্যন্ত ন্র, লাজুক, পর্দানশীল ও উত্তম চরিত্রের অধিকারিনী। তঁ|র সত্যবাদিতা, ন্যায়পরায়ণতা, কর্মদক্ষতা ও জন দরদি মনোভাব এলাকায় চাঞ্চল্যের সৃষ্টি করে। প্রকৃতপক্ষে তিনি সতী, সাধ্বী ও মহীয়সী নারী ছিলেন। তিনি বহু বিধর্মী নারীকে মুসলমান করেছেন। ২৫ বলা যায় তিনি তৎকালীন সময়ের একজন সুফি ছিলেন। কেননা পারিবারিক দিক থেকে সুফিবাদের সাথে তাঁর একটা ঘনিষ্ঠ যোগসূত্রের ঐতিহ্য রয়েছে। দুইভাবে সুফি হওয়া যায়। সাধনার মাধ্যনে এবং উত্তারাধিকার সূত্রে। মরিয়ম সালেহার পরিচয় সূত্রে জানা যায় যে, মরিয়ম সালেহার পিতা সৈয়দ আব্দুল্লাহ মোজাল্দেদী, পিতামহ সৈয়দ গোলাম মো. মোজাল্দেদী এবং ভাই সৈয়দ শাহ নূরী মুঘল আমলে বাংলার বিখ্যাত সুফি ছিলেন। তিনি বাবুপুরা নামক স্থানে গভীর বন জগলে খানকাহ্ স্থাপন করেন। তিনি লেখানেই 
সর্বময় আল্লাহর ধ্যানে মগ্ন থাকতেন আর বিধর্মীদের কাছে ইসলাম ধর্ম প্রচার করতেন। উপমহাদেশে তিনি ইসলাম ধর্ম প্রচার ও প্রসারের মাধ্যনে যথেষ্ট অবদান রেখেছেন। তাঁর ভাই শাহ নূরী বাবা ও দাদার মত ধর্মীয় কাজে নিযুক্ত ছিলেন বাবুপুরা এলাকায়। তৎকালীন সময়ে তিনি বাবুপুরা এলাকা থেকে মগবাজারের গভীর অরণ্যে খানকাহ প্রতিষ্ঠা করেন এবং সেখানে চলে যান। তৎকালে মুফতি, আলেম, ওলামাগণ তাঁর অনুমতিক্রমে ধর্মীয় বিষয়ে ফতোয়া প্রদান করতেন। তিনি ছিনেন অসাধারণ ধর্মীয় ব্যক্তিত্ব। তাঁর আধ্যাত্নিক মনোভাব এলাকাকে চাঙ্গা করে তুলেছিল। ২৬ শাহ নূরী মগবাজারে চলে গেলেও তার বড় বোন মরিয়ম সালেহা বাবুপুরা এলাকায় থেকে যান। এখানেই তিনি সর্বদা আল্লাহর ইবাদতে মগ্ন থাকতেন। নফল রোযা, নফল ইবাদত ছিল তার নিত্যদিনের ব্যাপার। তৎকালে তিনি মহিলাদের নিকট ধর্মীয় দাওয়াত প্রচার করতেন। এই মহীয়সী নারী মসজিদ নির্মাণাধীন অবস্থায় নামাজের মছলায় শেষ নিঃশ্বাস ত্যাগ করেন। তাঁর ইন্তেকালে এলাকায় বিরাট এক শোকের ছায়া নেনে আলে। অনেক দূর-দূরান্ত থেকে হাজারো মানুষ জানাজায় শরীক হন। ২৭ আর তারই নামানুসারে মসজিদটির নামকরণ করা হয় “মরিয়ম বিবি শাহী মসজিদ” । একই সাথে এটাও বুঝা যায় যে সমাজে সুফিদের প্রভাব ছিল। সুফি হিসেবে তাঁর ভক্তবৃন্দ তাঁকে অর্থ প্রদান করতেন এবং তিনি সেই অর্থ দিয়ে এই মসজিদ নির্মাণ করেছেন। ফলে বুঝা যায় যে, সমাজে তখন অর্থনৈতিক স্বচ্ছলতা বিরাজমান ছিল। কৃষি ও শিল্পজাত দ্রব্যের প্রাচুর্য এবং বিপুল পরিমাণ রপ্তানি বাণিজ্য মুঘল আমলে বাংলার সমৃদ্ধি ও সুলভ জীবনযাত্রায় সাহায্য করে। সমসাময়িক বিদেশি পর্যটক ও ব্যবাসায়ীগণ প্রদেশের সর্বত্র জিনিসপত্রের প্রাচুর্য ও সুলভ দাম দেখে অবাক হয়েছেন। চারটি জিনিলের দ্বারা একটি দেশের সমৃদ্ধি বিচার করা হয়: প্রথম, কৃষি ও শিল্পজাত দ্রব্যের প্রাচুর্য; দ্বিতীয়, অত্যাবশ্যকীয় জিনিসপত্রের সুলভতা; তৃতীয়ত, বিপুল আকারের বৈদেশিক বাণিজ্য এবং চতুর্থত, দেশে মূল্যবান পদার্থ, স্বর্ণ, রৌপ্য ইত্যাদি সঞ্চিত হওয়া। সমসাময়িক ফারসি ইতিহাস লেখকগণ ও ইউরোপীয় পরিব্রাজকগণের বর্ণনা থেকে প্রকাশ পায় যে, আর্থিক সমৃদ্ধির এই চারটি অত্যাবশ্যকীয় উপাদান মুঘল আমনে উল্লেখযোগ্য পরিমাণে বাংলায় বিদ্যমান ছিল। ২৮ গোলাম হোলেন সলিম লিখেছেন যে, সেকালে মাসিক মাত্র এক টাকা আয়ের উপর একজন লোক দৈনিক দুববার বেশ সুস্বাধু খাদ্য খেতে পারত। ২৯ মুঘল আমলে ঢাকার অর্থনৈতিক অবস্থা সম্পর্কে ড. আব্দুল করিম বলেন, “আমদানি, রপ্তানি, শহরের মধ্য দিয়ে পরিবাহিত মালামাল, ব্যবসায়ের উদ্দেশ্যে আগত বিপুল সংখ্যক দেশি ও বিদেশি বণিক, শহরে অবস্থানরত কর্মকর্তাগণ প্রভৃতি সবই শহরের অর্থনৈতিক সমৃদ্ধির ইংগিত প্রদান করে”। ৩০ আওরঙজেব আলমগীরের সময় থেকে মুঘল সম্রাটগণ এই প্রদেশের রাজস্ব ও করের উপর ব্যাপকভাবে নির্ভরশীল ছিলেন। বাবর বাংলার জনসাধারণ কর্তৃক স্বর্ণ ও রৌপ্য মজুদ করার অভ্যাসের উল্লেখ করেন। এতে প্রতীয়মান হয় যে, তাদের জীবন যাত্রার ব্যয়ভার নির্বাহ করার পর তাদের হাতে উদ্বৃত্ত সম্পদ থাকত।

তৃতীয়ত, আধ্যাত্নিকভাবে গুরুত্বপূর্ণ করার জন্য এই মসজিদের শিলালিপিতে ‘গুফত’ (ফারসি ভাষায় ‘গুফত’ অর্থ ‘বলা হচ্ছে’) শব্দ ব্যবহৃত হয়েছে। ফলে বুঝা যায়, সমাজে আধ্যাত্নিকতাবাদ প্রভাব রেখেছিল। কথিত আছে যে, মরিয়ম সালেহার পিতামহ হযরত গোলাম মোজাল্দেদী আধ্যাত্নিক ক্ষমতাপূর্ণ বুজর্গ ওলি ছিলেন। তৎকালীন হিন্দু সাধকদের সাথে তিনি অলৌকিকভাবে বিভিন্ন ধরনের কেরামতি দেখিয়ে অনেককে ইসলানের ছায়াতলে নিয়ে আলেন। ইতিহাস সাক্ষ্য দেয় যে, অসংখ্য জ্বীনের উস্তাদ ছিলেন তিনি। তিনি একবার দেয়ালের উপর বলে দেয়ালকে চলতে বললেন, ঠিক মুহূর্তের মধ্যে দেয়াল চলতে থাকল। ১১ মরিয়ম সালেহার ভাই শাহ নূরী (রহ.) এর সুযোগ্য পুত্র হযরত মাওলানা শাহ সৈয়দ গোলাম নোহাম্মদীও আধ্যাত্নিক ক্ষমতার অধিকারী ছিলেন। 
চতুর্থত, তৎকালে ফারসি ভাযা লিখার একটি শিল্পীগোষ্ঠী গড়ে উঠেছিল। যারা এ কাজে খুবই দক্ষ ছিল। কেননা, ভারতীয় উপমহাদ্লেশে মুঘল আমলে হস্তলিখন শিল্পের চরম উৎকর্ষ সাধিত হয়। সম্রাট বাবর হতে শুরু করে শেষ মুঘল সম্রাট বাহাদুর শাহ জাফর পর্যন্ত সব সম্রাট হস্তলিখন শিল্পে পারদর্শিতা অর্জন করেন। রাষ্ট্রীয় পৃষ্ঠপোষকতা লাভ করে সম্রাট আকবর, সম্রাট শাহজাহান এবং আওরঙ্গজেবের আমলে হস্তুলিখন শিল্পীগণ বিভিন্ন লিখন পদ্ধতি বিশেষ করে নাসতালিক পদ্ধতিতে পারদর্শিতা অর্জন করেন। প্রখ্যাত হস্তলিখন শিল্পীগণ সামাজিক ও রাষ্ট্রীয় মর্যাদা লাভ করে লিখন শিল্পকে বিভিন্নভাবে সুষমামঞ্তিত করে তুলেছিলেন। ৩২ তাছাড়া মুঘল-বাংলায় শিক্ষা বিস্তারে মুঘলদের পৃষ্ঠপোষকতা, সমাজে সরকারি কাজে ফারসির ব্যবহার আর জীবিকার জন্য হিন্দু-মুসলিম উভয়রেই ফারসি ভাষা শিখার বাধ্যবাধকতা প্রভৃতি সমকালীন বাংলার শিক্ষা ও শৈল্পিক মান উন্নত করনে সাহায্য করে। ৩০ পঞ্চমত, আরবি তুঘরা রীতির পরিবর্তে শিলালিপিতে পার্সিয়ান, নাসতালিক রীতির ব্যবহার এটাই প্রমাণ করে যে, ঐ সময়ে পারস্য সভ্যতার সাথে মুঘল বাংলার ঘনিষ্ঠ সম্পর্ক ছিল।

ষষ্ঠত, রাষ্ট্রীয় পৃষ্ঠপোষকতার বাইরে স্থানীয়ভাবে নির্মাণের একটি নিদর্শন এই শিলালিপিটি। এতে বুঝা যায় এরকম আরো অনেকে এ ধরনের নির্মাণ কাজ করত।

সপ্তমত, শিলালিপিটিতে নির্মাণ সন স্পষ্টতভাবে উল্লেখ আছে ১১১৮- হিজরি (১৭০৬ খ্রি.)। অর্থাৎ বুঝা যাচ্ছে সম্রাট আওরঙজেবের সময় নির্মিত এটি একটি আদর্শ মুঘল মসজিদ।

\section{উপসংহার}

উপরোক্ত সার্বিক আলোচনার প্রেক্ষিতে বলা যায় যে, ইতিহাস অন্বেষণে তথা এর গবেষণায় শিলালিপি ব্যাপকতর গুরুত্বে পরিণত হয়েছে। ইতিহাসের উৎস হিসেবে শিলালিপির ব্যবহারে ইতিহাসের রূপরেখা অনেকটা সুস্পষ্ট হয়ে উঠে। কেননা মধ্যযুগের মুঘল বাংলার ইতিহাস পুনর্গঠনে শাসকদের পৃষ্ঠপোষকতায় রচিত ইতিহাস গ্রন্থগুলোতে রাজনৈতিক ইতিহাস যতোটা প্রাধান্য পেয়েছে সমকালীন জনসাধারণের আর্থ-সামাজিক, সাংস্কৃতিক ও ধর্মীয় অবস্থার চিত্র ততোটাই উপেক্ষিত হয়েছে। তাই রাজনৈতিক ইতিহাসের বাইরে মুঘল বাংলার বিভিন্ন দিক যেমন সামাজিক, সাংস্কৃতিক কর্মকাঞ, অর্থনৈতিক জীবন, ধর্মীয় অবস্থার ইতিহাস রচনায় শিলালিপি আলাদা অবস্থান করে নিতে সক্ষম হয়েছে এবং ইতিহাস রচনায় এসব লিপিসাক্ষ্য বিশ্লেষণ অপরিহার্য হয়ে পড়েছে। আলোচ্য প্রবন্ধের হাজী খাজা শাহবাজ ও মরিয়ম সালেহা মসজিদের শিলালিপি ${ }^{34}$ দু’টির বিশ্লেষণধর্মী আলোচনা প্রকৃত অর্থে এ বিষয়ে সকল বিশেষজ্ঞগণের দৃষ্টি আকর্ষণের এটি একটি ক্ষুদ্র প্রয়াস।

\section{References}

1. Rahim, M. A. (2012). Banglar Samajik O Sangskrititk Itihas, Bangla Academy, Dhaka, p. 37.

2. Karim, A. (1977). Banglar Itihas (Sultani Amol), Bangla Academy, Dhaka, p. 34.

3. Hossain, A. B. M. (ed.). (2007). Sthapotto, Asiatic Society of Bangladesh, p. 276.

4. Karim, A. (1992). Corpus of the Arabic and Persian inscriptions of Bengal, Asiatic Society of Bangladesh, p. 470.

5. Sompadok Mondoli. (2003). Banglapedia, $3^{\text {rd }}$ Vol. Asiatic Society of Bangladesh, Dhaka, p. 347. 
6. Karim, A. (2003). Mogal Rajdhani Dhaka, Bangla Academy, Dhaka, p. 55-56.

7. Travels of Sebastian Manrique, Vol-I, Pp. 44-45.

8. Islam, M. M. and Mahmud, F. (ed.). (2012). Rajdhani Dhakar 400 Bochor O Uttarkal, 2 Khondo, Bangladesh Asiatic Society, Dhaka, p. 35.

9. Khan, M. M. (Trans. and ed.). (Hijri-1413). Tafsir Mareful Quran, Khademul Haramain Shorifain Badshah Fahd Qur'an Mudron Prokolpo, Modina Monoara, p. 1411.

10. Sohih Bukhari Sharif. (n.d.). Subject: Namaj, Poricched-306, Hadith No. 437, Vol. I, Chapter Seven, Islamic Foundation, Dhaka, Pp. 246-247.

11. Sohih Muslim. (1999). Chapter Five, Paragraph Four, Hadith No. 1079, Bangladesh Islamic Center, Dhaka, p. 299.

12. Islam, S. (ed.). (2003). Bangladesher Itihas, Asiatic Society of Bangladesh, Dhaka, p. 3.

13. Karim, A. (1992). Corpus of the Arabic and Persian inscriptions of Bengal, Asiatic Society of Bangladesh.

14. Ibid.

15. Rahim, M. A. (2012). Banglar Samajik O Sangskrititk Itihas, Bangla Academy, Dhaka, Pp. 15-16.

16. Jakaria, A. K. M. (2011). Bangladesher Protnosompod, Dibboprokash, Pp. 538-539.

17. Ahmed, S. (1960). Inscriptions of Bengal, Vol. IV, Rajshahi.

18. Karim, A. Ibid.

19. Dani, A. H. (1962). Dacca: A Record of Its Changing Fortunes, Dacca, p. 212.

20. Siddiqi, M. Y. (n.d.). Arabic and Persian Text of the Islamic Inscription of Bengal, South Asia Press, Massachusetts 02172-3016, USA.

21. The Dhaka University Studies. (1997). Vol. 54, No. 2.

22. Rahman, H. H. (1990). Asudgan-e-Dhaka, Onubad-Mowlana Akram Faruq O Mowlana A. N. M. Ruhul Amin Chowdhury, Pp. 53-54.

23. Abdullah, M. (1991). Dhakar Koyekjon Muslim Sudhi, Islamic Foundation Bangladesh, p. 273.

24. Bongo Bichitra. (2006). Dhaka: Sonibar, 16-22 December, 2-8 Poush, 1413, p. 6.

25. Bongo Bichitra. (2006). Dhaka: Sonibar, 16-22 December, 2-8 Poush, 1413, p. 5.

26. Ibid.

27. Ibid.

28. Rahim, M. A. Ibid, p. 314.

29. Uddin, A. (Trans.). (1974). Riaj-us-salatin. Dhaka, Bangla Academy, p. 304.

30. Karim, A. (2003). Mogal Rajdhani Dhaka, Bangla Academy, p. 75.

31. Bongo Bichitra. (2006). Dhaka: Sonibar, 16-22, December, 2-8 Poush, 1413, p. 5.

32. Ali, A. K. M. Y. (2010). Muslim Mudra O Hostolikhon Shilpo, Bangla Academy, Pp. 308-309. 
33. Islam, M. M. and Mahmud, F. (ed.). (2012). Rajdhani Dhakar 400 Bochor O Uttarkal, Ditio Khondo, Bangladesh Asiatic Societyt, p. 119.

34. Gobeshokbrindo 2015 soner 12 abong 15 July Sorejomine Shilalipigulo Dekechen.

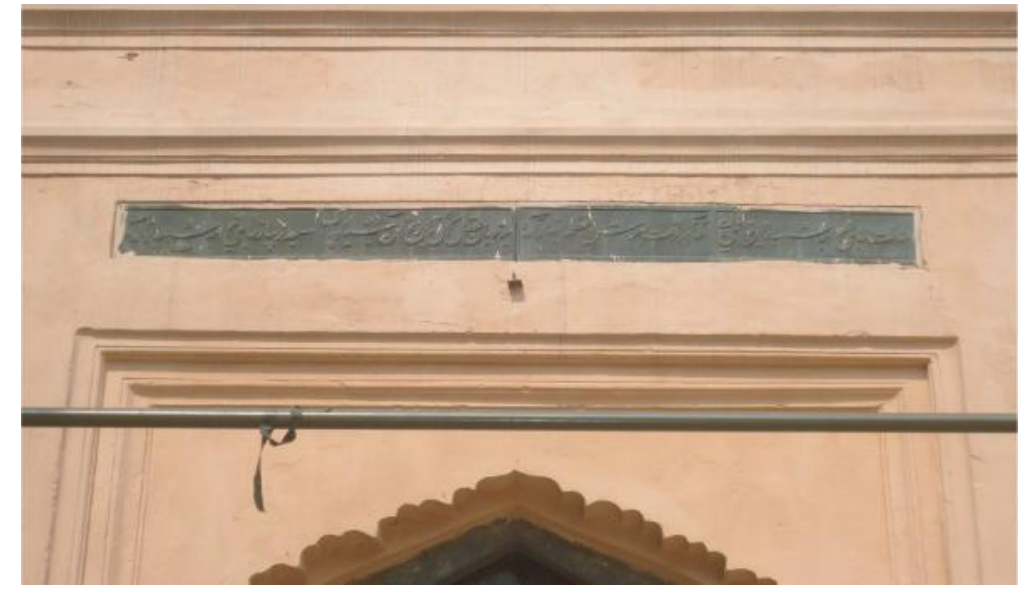

চিত্র: ১ (হাজী খাজা শাহবাজ মসজিদের শিলালিপি) ছবি: গবেষক ২০১৫ সনের ১২ এবং ১৫ জুলাই সরেজমিনে পর্যবেক্ষণ করে নিজ ক্যামেরায় ছবিটি তুলেছেন।

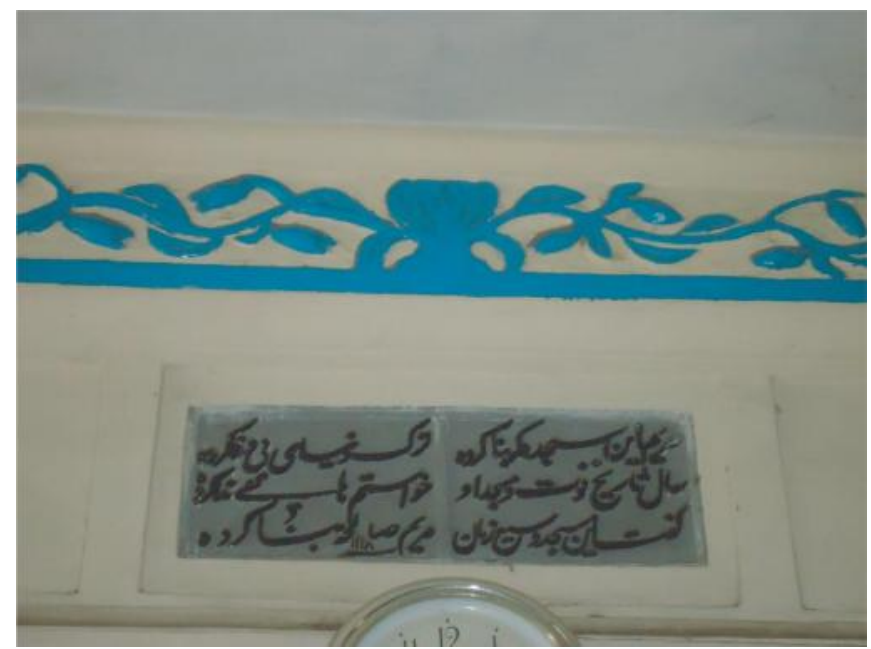

চিত্র: ২ (মরিয়ম সালেহা মসজিদের শিলালিপি) ছবি: গবেষক ২০১৫ সনের ১২ এবং ১৫ জুলাই সরেজমিনে পর্যবেক্ষণ করে নিজ ক্যানেরায় ছবিটি তুলেছেন। 